\title{
Collagen Peptide Presenting Nanofibrous Scaffold for Intervertebral Disc Regeneration
}

\author{
Ozge Uysal, ${ }^{\dagger, \dagger}$ Elif Arslan, ${ }^{\dagger, \S}$ Gulcihan Gulseren, ${ }^{\dagger, \S}$ Mustafa Cemil Kilinc, ${ }^{\perp}$ Ihsan Dogan, ${ }^{\perp}$
} Hakan Ozalp, ${ }^{\text {II }}$ Yusuf Sukru Caglar, ${ }^{*, \perp}$ Mustafa O. Guler, ${ }^{*}{ }^{\#} \odot$ and Ayse B. Tekinay, ${ }^{*},,+, \xi_{\odot}$

${ }^{\dagger}$ Institute of Materials Science and Nanotechnology, National Nanotechnology Research Center (UNAM), ${ }^{+}$Neuroscience Graduate Program, and ${ }^{\S}$ Materials Science and Nanotechnology Graduate Program, Bilkent University, Ankara 06800, Turkey

${ }^{\perp}$ Department of Neurosurgery, Ankara University Faculty of Medicine, Ankara 06100, Turkey

${ }^{\mathbb{I}}$ Department of Neurosurgery, Mersin University Faculty of Medicine, Mersin 33343, Turkey

${ }^{\#}$ Institute for Molecular Engineering, University of Chicago, Chicago, Illinois 60637, United States

\section{Supporting Information}

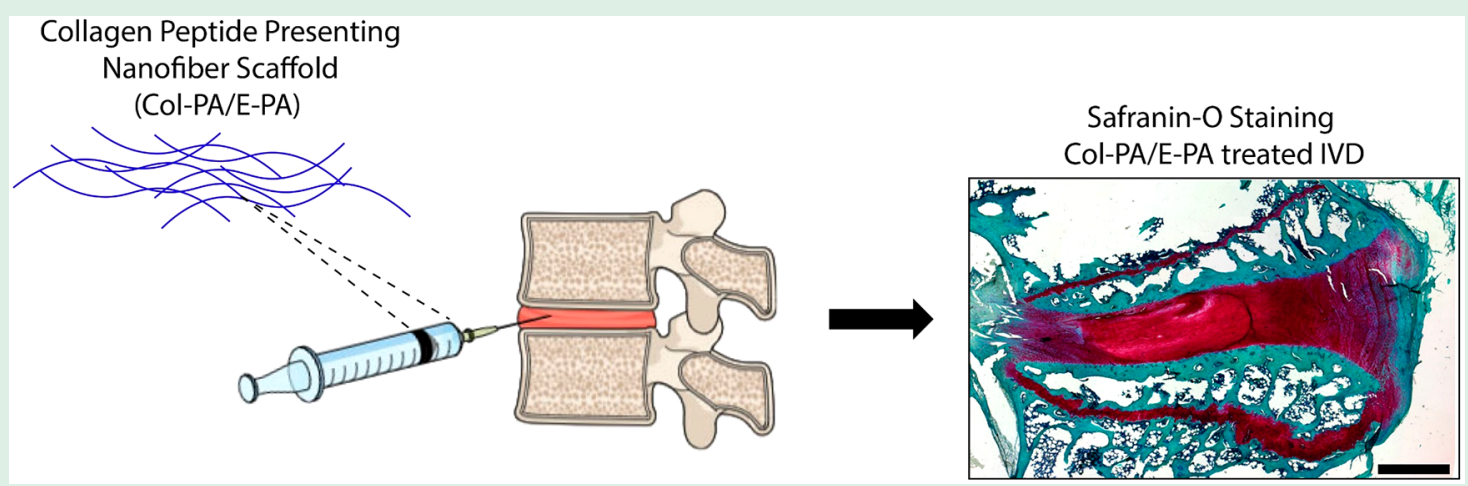

ABSTRACT: Lower back pain (LBP) is a prevalent spinal symptom at the lumbar region of the spine, which severely effects quality of life and constitutes the number one cause of occupational disability. Degeneration of the intervertebral disc (IVD) is one of the well-known causes contributing to the LBP. Therapeutic biomaterials inducing IVD regeneration are promising candidates for IVD degeneration treatments. Here, we demonstrate a collagen peptide presenting nanofiber scaffold to mimic the structure and function of the natural extracellular matrix of the tissue for IVD regeneration. The collagen peptide presenting nanofiber was designed by using a Pro-Hyp-Gly (POG) peptide sequence on a self-assembling peptide amphiphile molecule, which assembled into nanofibers forming scaffolds. Injection of collagen peptide presenting peptide nanofiber scaffold into the degenerated rabbit IVDs induced more glycosaminoglycan and collagen deposition compared to controls. Functional recovery of the tissue was evaluated by degeneration index score, where the bioactive scaffold was shown to provide functional recovery of the IVD degeneration. These results showed that the collagen peptide presenting nanofiber scaffold can prevent the progression of IVD degeneration and provide further functional recovery of the tissue.

KEYWORDS: peptide nanofiber, intervertebral disc degeneration, needle puncture model, regeneration, collagen

\section{INTRODUCTION}

Lower back pain (LBP) is a prevalent condition that many people experience ${ }^{1,2}$ and, together with neck problems, the most common spinal disorder especially in aging populations. ${ }^{3}$ The main cause of the LBP is the degeneration of the intervertebral discs (IVDs), which reside between two vertebrae. IVD has a fibrocartilage structure and provides flexibility and stability to the spinal column. ${ }^{4}$ IVD degeneration can be caused due to lifestyle, obesity, lack of physical activity, or genetic background of the patients. ${ }^{5}$ The IVD tissue consists of nucleus pulposus (NP), annulus fibrosus (AF), and cartilaginous end plates. The NP forms the inner part of the tissue and comprises randomly organized collagen fibers (mainly collagen type-II) and radially arranged elastin fibers, which are embedded in a hydrated aggrecan-rich gelatinous structure. ${ }^{6,7} \mathrm{NP}$ is surrounded by AF, which consists of highly oriented collagen type-I forming lamellae structures. ${ }^{8}$ The cartilage end-plates, which are composed of hyaline cartilage and osseous components, lie between the vertebral body and the disc. In all of these three structures, chondrocyte- and fibroblast-like cells are present predominantly., ${ }^{9,10}$ In addition to these cellular components, acellular macromolecules such as glycosaminoglycans, proteoglycans, and collagens constituting the extracellular matrix (ECM) are essential for the proper

Received: January 24, 2019

Accepted: March 18, 2019

Published: March 18, 2019 


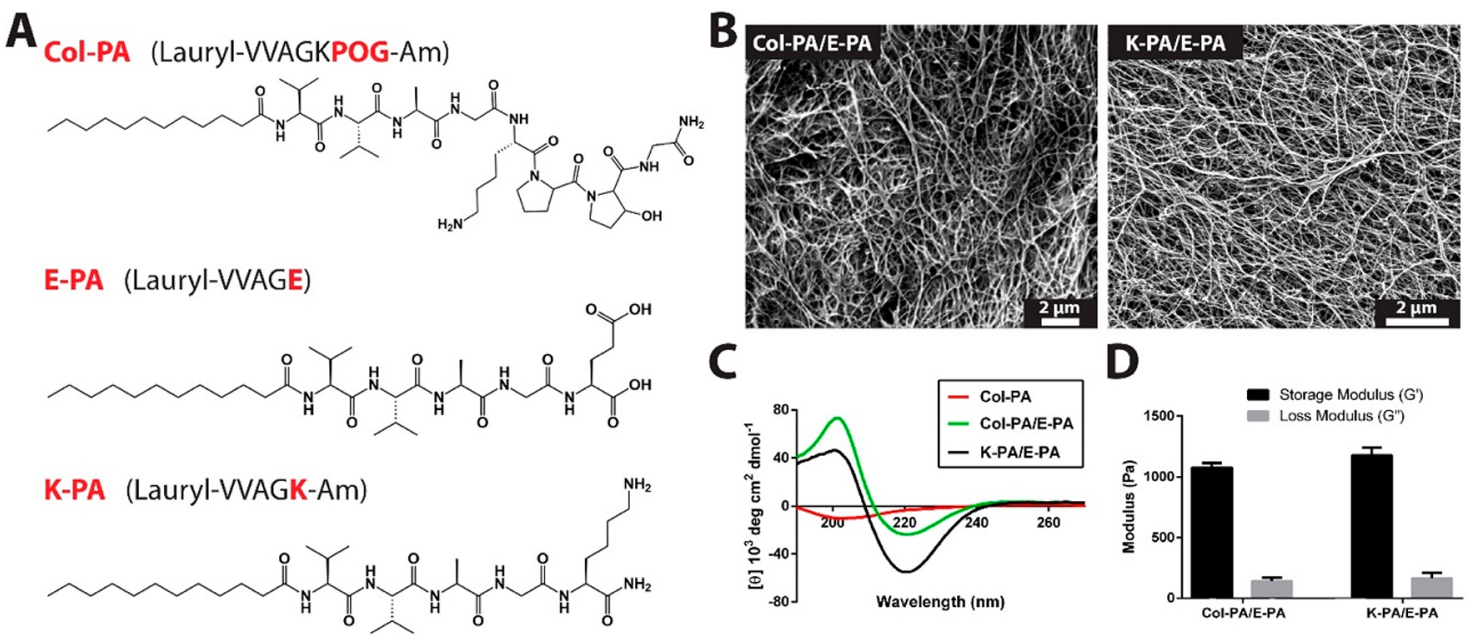

Figure 1. Peptide molecules and their characterizations. (A) Chemical structures of Col-PA, E-PA, and K-PA. (B) Structural characterization of the peptide nanofibers at $\mathrm{pH}=7.4$ by SEM. (C) Circular dichroism spectra of Col-PA, Col-PA/E-PA, and K-PA/E-PA. (D) Rheological analyses of peptide nanofibers.

functioning of the tissue. ${ }^{11}$ The changes in the composition of the IVD tissue may result in severe mechanical consequences leading to diseases. The current methods for treatment of IVD degeneration provide limited long-term solutions. ${ }^{12-14}$ Since the main reason behind the IVD degeneration is degradation of the ECM components such as aggrecan and collagen, effective treatment strategies should promote the regeneration of the tissue by inducing chondrocyte differentiation and ECM remodelling in the degenerated tissue. Therefore, novel therapeutics such as ECM mimetic biomaterials can be utilized for efficient treatment of IVD degeneration.

Several treatment methods have previously been studied for IVD regeneration including implantation of NP cells grown on atelocollagen scaffold to a rabbit IVD degeneration model. ${ }^{2}$ In another study, NP cells cultured on collagen-I/hyaluronan scaffold were shown to induce cell proliferation and glycosaminoglycan synthesis; however, most of the secreted glycosaminoglycans were released to the cell culture media rather than being retained in the scaffold. ${ }^{15}$ For all potential methods for the treatment of IVD degeneration, the viability and proliferation of the resident disc cells and the surrounding ECM of the tissue will determine the efficacy of the treatment. $^{16}$

In this work, we demonstrate a synthetic peptide nanofiber hydrogel as a scaffold to mimic ECM for IVD regeneration. The peptide nanofiber systems provide useful tools to produce biocompatible, nonimmunogenic, and aqueous environments for the cells in NP, which has a very high-water content in its healthy state. ${ }^{11,17,18}$ The self-assembling peptides can be functionalized with bioactive epitopes present in the ECM of specific tissues such as muscles, bones, or neural tissues. ${ }^{19-24}$ Here, a collagen sequence presenting peptide amphiphile molecule was designed by using Pro-Hyp-Gly (POG) artificial collagen sequence ${ }^{25}$ along with a self-assembling short peptide sequence (Lauryl-VVAGK-Am, K-PA) to create a collagen mimetic structure. ${ }^{26}$ The Col-PA (Lauryl-VVAGKPOG-Am) and E-PA (Lauryl-VVAGE) peptide nanofibers were used to create a de novo collagen mimetic microenvironment (Col-PA/ E-PA nanofiber gel) (Figure 1). The Col-PA/E-PA nanofiber gel facilitated the recovery of degeneration of cartilage-like IVD tissue. The in vitro efficacy of the Col-PA/E-PA nanofibers was shown through viability analysis, glycosaminoglycan deposition analysis, and gene expression analysis by using mesenchymal stem cells (MSCs). The in vivo IVD regeneration in the rabbit needle puncture model was monitored by histochemical analysis, and evaluation was performed for 4 weeks of recovery. The Col-PA/E-PA treatment enhanced deposition of glycosaminoglycans and collagen and resulted in a decrease in the degeneration score compared to the control groups, which strongly suggested that Col-PA/E-PA nanofiber scaffold can be utilized as an effective treatment method for IVD degeneration.

\section{EXPERIMENTAL SECTION}

Materials. Fmoc (9-fluorenylmethoxycarbonyl) and Boc (tertbutoxycarbonyl) protected amino acids, lauric acid, Rink amide MBHA resin, Fmoc-Asp(OtBu)-Wang resin, HBTU (2-(1H-benzotriazol-1-yl)-1,1,3,3-tetramethyluronium hexafluorophosphate), and DIEA (diisopropylethylamine) were purchased from NovaBiochem and $A B C R$. All other chemicals and materials were obtained from Invitrogen, Fisher, Merck, Alfa Aesar, and Sigma-Aldrich.

Synthesis and Purification of Peptide Amphiphile Molecules. Fmoc solid phase peptide synthesis procedure was used for amphiphilic peptides. Col-PA molecule was synthesized on MBHA Rink Amide resin at $0.25 \mathrm{mmol}$ scale. Amino acid couplings were performed with 2 equiv of Fmoc-protected amino acid, 1.95 equiv of HBTU, and 3 equiv of DIEA for $2 \mathrm{~h}$. The $20 \%$ piperidine/ dimethylformamide (DMF) solution was used for Fmoc removal on the amino group. A mixture of trifluoroacetic acid (TFA)/ triisopropylsilane (TIS) $/ \mathrm{H}_{2} \mathrm{O}$ in the ratio of $95: 2.5: 2.5$ for $3 \mathrm{~h}$ was used for cleavage of the peptides from the resin. Peptides were HPLC purified and purity and molecular weight were confirmed by LC-MS analyses (Figure S1).

Scanning Electron Microscopy (SEM). Coverslip surfaces were coated with mixtures of $10 \mathrm{mM}$ Col-PA with $10 \mathrm{mM}$ E-PA or $10 \mathrm{mM}$ K-PA with $10 \mathrm{mM}$ E-PA peptide solutions and incubated for $15 \mathrm{~min}$ for gel formation. Critical point drying procedure was applied for water removal. Environmental scanning electron microscope (FEI Quanta 200 FEG, 10-15 kV voltage) was used for SEM imaging. Coverslips were coated with $3 \mathrm{~nm} \mathrm{Au}-\mathrm{Pd}$ prior to imaging.

Circular Dichroism (CD). Peptide solutions were dissolved in water. Both Col-PA and K-PA solutions were mixed with E-PA solution and were incubated for $15 \mathrm{~min}$ to form nanofibers. The samples were diluted with water to a concentration of $1 \times 10^{-4} \mathrm{M}$. Parameters for CD measurements were set at DIT $4 \mathrm{~s}$, bandwidth $1.00 \mathrm{~nm}$, data pitch $0.2 \mathrm{~nm}$, start mode $1 \mathrm{~mm}$, and scanning mode continuous. 
Oscillatory Rheology Analyses of Materials. Oscillatory rheology (Anton Paar Physica RM301) measurement was performed for determining the viscoelastic properties of nanofiber gels. A $25 \mathrm{~mm}$ parallel plate was used for the rheometer at $25{ }^{\circ} \mathrm{C}$. Then $125 \mu \mathrm{L}$ of each PA sample (Col-PA, E-PA, and K-PA) was used. The peptide gels (Col-PA/E-PA and K-PA/E-PA) at 1 wt $\%$ concentration were loaded on the center of the lower plate and incubated for $15 \mathrm{~min}$ before measuring. After equilibration, the upper plate was lowered to a gap distance of $0.5 \mathrm{~mm}$. Under $100 \mathrm{rad} / \mathrm{s}$ to $0.1 \mathrm{rad} / \mathrm{s}$ of angular frequency, with a $0.5 \%$ shear strain, storage moduli $\left(G^{\prime}\right)$ and loss moduli $\left(G^{\prime \prime}\right)$ were measured.

In Vitro Cell Culture Experiments. Cell culture experiments were performed with rat mesenchymal stem cells at passage 3 (postthaw (rMSC, Invitrogen S1601-100). According to the manufacturer's (Invitrogen) instructions, the cells maintained stem cell proliferation at post-thaw passage number 5 . Cells were cultured with DMEM supplemented with $10 \%$ fetal bovine serum (FBS), $1 \%$ GlutaMAX, and $1 \%$ penicillin-streptomycin (Maintenance Media) and were incubated in the humidified atmosphere with $5 \% \mathrm{CO}_{2}$ at 37 ${ }^{\circ} \mathrm{C}$. For the differentiation analysis, cells were cultured with chondrogenic differentiation media (Gibco).

For the in vitro experiments, peptide amphiphile molecules were dissolved in $\mathrm{ddH}_{2} \mathrm{O}$ at a concentration of $1 \mathrm{mM}$. After $30 \mathrm{~min}$ of sonication, they were sterilized under UV for $1 \mathrm{~h}$. Then well plates (96-well plates or 48-well plates) were coated with $1 \mathrm{mM} \mathrm{Col-PA}$ and $1 \mathrm{mM}$ E-PA at a 1:1 ratio for the Col-PA/E-PA group and $1 \mathrm{mM} \mathrm{K}$ $\mathrm{PA}$ and $1 \mathrm{mM}$ E-PA at a 1:1 ratio for the K-PA/E-PA group. These peptide-coated plates were dried at room temperature overnight for the hydrogel formation. On the next day, cells were seeded at 3000 cells $/ \mathrm{cm}^{2}$. As a control, tissue culture plate (TCP) was used.

Cell Viability Analysis and Glycosaminoglycan Staining. Cell viability was determined by alamarBlue and Live/Dead kits on day 3 of culture by quantifying both absorbance and fluorescence of the reagent using spectrophotometry for alamarBlue assay and imaging the cells by fluorescent microscopy for the Live/Dead assay.

Glycosaminoglycan deposition was assessed by safranin-O staining at the end of day 7 and day 14. Briefly, after the removal of culture medium, cells were washed with PBS and then fixed with $4 \%$ paraformaldehyde for $15 \mathrm{~min}$ at room temperature. After the fixation, cells were blocked with $1 \%$ BSA in PBS for $1 \mathrm{~h}$. Cells were then stained with $0.1 \%$ safranin-O in $0.1 \%$ acetic acid for $5 \mathrm{~min}$ at room temperature. To remove unspecific binding of the safranin-O dye, cells were extensively washed with PBS three times, and they were observed by light microscopy.

Gene Expression Analysis. For analyzing chondrogenic differentiation, gene expression profile of Sox9 gene was assessed by quantitative real-time PCR (qRT-PCR). RNA from the cells that were cultured on the peptide nanofibers and tissue culture plate was isolated with TRIzol reagent (Invitrogen) according to the manufacturer's instructions. Purity and yield of the samples were determined with Nanodrop 2000 (Thermo Scientific). By using a one-step qRT-PCR kit (SuperScript III Platinum SYBR Green), amplification of both cDNA and mRNA was performed according to the manufacturer's instructions. In summary, the reaction was performed as follows: $55^{\circ} \mathrm{C}$ for $5 \mathrm{~min}, 95{ }^{\circ} \mathrm{C}$ for $5 \mathrm{~min}, 40$ cycles of $95{ }^{\circ} \mathrm{C}$ for $15 \mathrm{~s}, 61.5{ }^{\circ} \mathrm{C}$ for $30 \mathrm{~s}$, and $40{ }^{\circ} \mathrm{C}$ for $1 \mathrm{~min}$. After the reaction, melting curve analysis was performed to confirm the product specificity. The expression level of Sox 9 was analyzed at days 7 and 14. The gene expression data were normalized to the expression levels of GAPDH in each run as an internal control gene and were analyzed by using comparative $\mathrm{Ct}$ method.

In Vivo Intervertebral Disc Degeneration with Needle Puncture Model in Rabbits and Applications of Scaffolds. Animal studies were performed in the animal facility of Ankara University Faculty of Medicine Ibni Sina Hospital, Department of Neurosurgery and Animal Laboratory. Protocols for animal care and handling were approved by Ankara University Animal Experimentation Ethics Committee with the ethical approval number as 201522-239. Eighteen healthy, $2.5-3 \mathrm{~kg}$ male New Zealand rabbits were used in this study ( $n=3$ per group) to avoid any effects resulting from hormonal fluctuations of female rabbits. Rabbits were housed in standard cages in a temperature and light controlled environment (16-22 ${ }^{\circ} \mathrm{C}$ and $12 / 12 \mathrm{~h} \mathrm{light/dark}$ cycles). Throughout the experiment, they were allowed ad libitum access food and water.

The rabbit model of IVD degeneration was created by the annulus needle puncture technique through a posterolateral retroperitoneal approach. Following anesthesia with an intramuscular injection of ketamine $(20 \mathrm{mg} / \mathrm{kg})$ and xylazine $(2 \mathrm{mg} / \mathrm{kg})$, animals were positioned in their lateral decubitus with 20 degrees. A longitudinal skin incision was performed to paraspinal muscles that lie from rib cage to pelvic rim. To reach the IVD, retroperitoneal adipose and muscle tissues were incised between L1 and L5 vertebrae. L5-L6 level of the IVD was determined according to landmarks of the lumbar interspinous and pelvic rims. Once the two levels of IVDs of interests (L3-4 and L4-5) were identified, an 18-gauge (18G) needle was inserted into the left anterolateral annulus fibrosus and held in the tissue for $5 \mathrm{~s}$. After the indicated degeneration period, these disc levels were injected with either peptide amphiphile solutions or saline solution. L2-3 and L5-6 disc levels were used as a healthy control. For one group of the animals (immediate degeneration), gel injection was performed immediately after the degeneration procedure, whereas, for the other group (delayed degeneration), the same region was opened again after 2 weeks of the degeneration process and peptide nanofiber injection was performed by insulin needle. The gels that were injected are summarized in Table S1. For the in vivo experiments, $10 \mathrm{mM}$ peptide amphiphile solutions were mixed at a 1:1 ratio, and in a final volume of $60 \mu \mathrm{L}$, peptide nanofiber mixture was injected into the degenerated disc space. Four weeks after the gel injections, animals were sacrificed and tissue regeneration was examined by histopathological examinations of IVD tissue.

IVD Cell Isolation from Rats. IVD cells were isolated as discussed in Lee et al. ${ }^{24}$ Briefly, IVD tissues were dissected from two rats and placed into IX PBS. Here, pieces of IVD tissues were finely diced with a blade and then incubated with $0.05 \%$ collagenase in DMEM+Ham's F-12 medium supplemented with $15 \%$ FBS for the ECM digestion process for overnight at $37{ }^{\circ} \mathrm{C}$. After overnight incubation, pipetting was done for further digestion of ECM molecules. Collagenase containing residual IVD tissues was retrieved, and cell solution was filtered by using a cell strainer to remove tissue debris. After filtering, cell solution was centrifuged at $2500 \mathrm{rpm}$ for 5 $\mathrm{min}$. Then cells were seeded into culture medium at a density of $2.5 \times$ $104 \mathrm{cells} / \mathrm{cm}^{2}$.

Histological and Immunohistochemical (IHC) Staining of Tissue Sections. After the extraction of tissues, samples were fixed in $4 \%$ paraformaldehyde for $48 \mathrm{~h}$ at room temperature and then decalcified in 5\% formic acid. Every 2 days, the formic acid solution was changed, and this process was continued for about 10-14 days. This process was ended according to the result of the ammonium oxalate test. Dehydrated and decalcified samples were by immersing in ethanol solutions and then were cleared in two changes of xylene. Samples were embedded in paraffin blocks and sectioned at 5-7 $\mu \mathrm{m}$ thickness by using a microtome. For the histological and immunohistological analyses, tissue sections were deparaffinized in two changes of xylene and then rehydrated by immersing in a series of ethanol solutions of decreased concentrations. Defect healing was determined by the staining of representative slides taken at every 20 sections $(\sim 100 \mu \mathrm{m}$ intervals $)$ to demonstrate the extent of IVD regeneration.

Safranin-O and Alcian Blue staining protocols were performed for imaging the glycosaminoglycan content of the IVD tissues. For safranin-O staining, tissues were stained with safranin-O for $1 \mathrm{~min}$ and Fast Green for $5 \mathrm{~min}$. For Alcian blue staining, tissues were stained with Alcian blue for 30 min and counterstained with nuclear Fast Red solution for $5 \mathrm{~min}$. Masson's trichrome and Picro Sirius red stainings were done to determine the content of collagen in the regenerated tissues. For Masson's trichrome staining, the samples were stained with hematoxylin for $45 \mathrm{~s}$, Biebrich scarlet for $5 \mathrm{~min}$, phosphomolybdic acid/phosphotungstic acid mixture for $5 \mathrm{~min}$, and aniline blue for $5 \mathrm{~min}$. For Picro Sirius red staining, tissues were stained with 

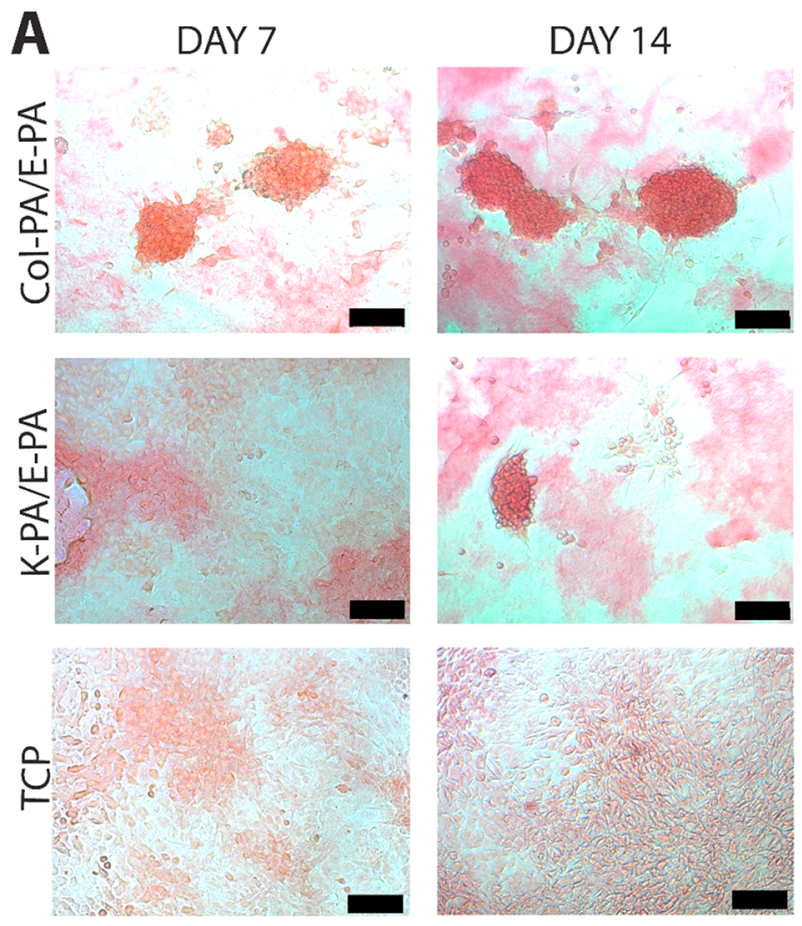

B
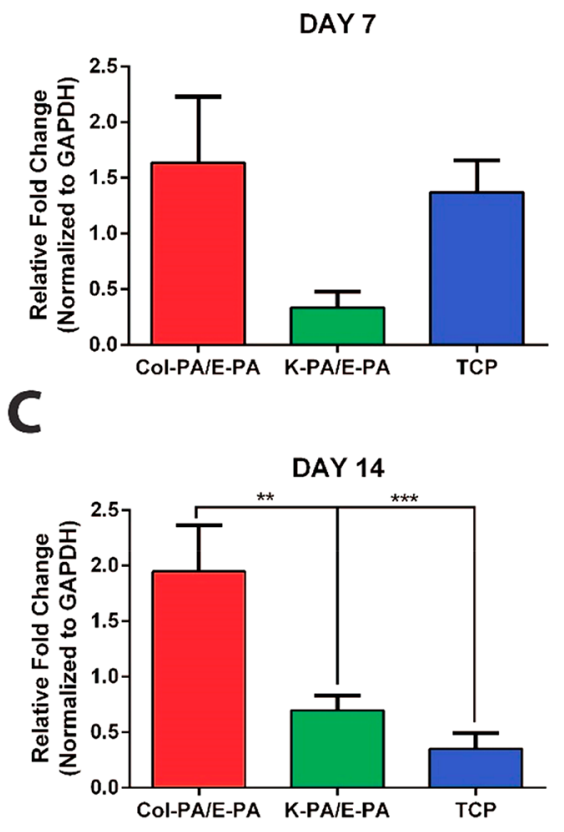

Figure 2. In vitro chondrogenic differentiation analyses of MSCs. (A) Representative images of safranin-O staining of MSCs cultured on peptide nanofibers and TCP in the chondrogenic media $($ scale bars $=100 \mu \mathrm{m})$. Chondrogenic differentiation analyses by qRT-PCR. Expression level of Sox9 was quantified on (B) day 7 and (C) day $14(* * p=0.0071, * * * p=0.0010$ by one-way ANOVA with Tukey post-test, mean \pm s.e.m.).

Weigert's hematoxylin for $8 \mathrm{~min}$ and then Picro Sirius red for $1 \mathrm{~h}$ to visualize the collagen fiber orientations. After the staining procedures, slides were dehydrated in a series of ethanol solutions of increasing concentrations and were cleared with xylene before mounting with Histomount mounting medium and were imaged under light microscopy.

For the antigen retrieval process of immunohistochemical staining, slides were treated with pepsin at $37{ }^{\circ} \mathrm{C}$ for $10 \mathrm{~min}$, and to block endogenous peroxidase activity, slides were treated with $0.3 \%$ hydrogen peroxide solution for $45 \mathrm{~min}$ at room temperature. After the blocking with $10 \%$ normal goat serum in $1 \%$ bovine serum albumin/Tris-buffered saline, slides were incubated with primary antibody against collagen type-II (MA-13026) at $4{ }^{\circ} \mathrm{C}$ overnight. After the washing steps with $0.01 \%$ triton-X in Tris-buffered saline, slides were incubated with HRP conjugated goat antimouse $\operatorname{IgG}$ antibody for $1 \mathrm{~h}$ at room temperature and incubated with 3,3'diaminobenzidine solution for $20 \mathrm{~min}$ to visualize collagen type-II proteins. Slides were then counterstained with hematoxylin for $45 \mathrm{~s}$ to visualize cell nuclei.

Histological Scoring. For histopathological assessment of IVD degeneration, degenerative changes in both NP and AF in sagittal sections of the disc were graded using the grading scale described by Nagae et al., ${ }^{27}$ which is briefly described in Table S2. All gradings were performed by blind observers who were not familiar with the animal groups and treatments. Scoring method for NP degeneration was done by analyzing hematoxylin and eosin stained sections to better observe cellular content, whereas scoring method for $\mathrm{AF}$ degeneration was done by using safranin-O stained sections to observe the general structure of the tissue.

Statistical Analysis. All results are represented as mean \pm s.e.m. (standard error of the mean). Student's $t$ test and one-way ANOVA with Tukey's post-test where appropriate were used to show the significance of the differences between the groups. $P<0.05$ was used to determine the significance of differences.

\section{RESULTS}

Synthesis and Characterization of Peptide Nanofiber Systems. Collagen sequence presenting peptide amphiphile molecule (Col-PA) was designed by addition of the active domain of the well-known artificial collagen sequence, POG, to a $\beta$-sheet forming VVAG sequence and a hydrophobic lauryl tail (Figure 1A). The Col-PA was mixed with negatively charged E-PA molecule at equal volumes to initiate selfassembly through charge neutralization. Through self-assembly process, Col-PA monomers are aligned in a close proximity in the nanofiber system and the collagen mimetic epitopes are presented on the peptide nanofiber scaffold. As the control nanofiber, the peptide amphiphile molecules, which does not have any bioactive sequence (positively charged K-PA and negatively charged E-PA molecules) were mixed at equal volumes. The porous network formed by the peptide nanofibers was observed by SEM (Figure 1B). The secondary structures of the peptide nanofibers were characterized by using CD spectroscopy, which demonstrated that both bioactive and control nanofibers exhibited $\beta$-sheet structure as shown by $200 \mathrm{~nm}$ positive and $220 \mathrm{~nm}$ negative signals (Figure 1C). Mechanical properties of the peptide nanofiber networks were investigated by oscillatory rheology. Both nanofiber Col-PA/E-PA and K-PA/E-PA nanofiber networks had higher storage modulus (for Col-PA/E-PA $1041 \mathrm{~Pa}$ and for K-PA/E-PA $1110 \mathrm{~Pa}$ ) values than loss modulus (for ColPA/E-PA $158 \mathrm{~Pa}$ and for K-PA/E-PA $117 \mathrm{~Pa}$ ) values and showed stable and robust mechanical properties as hydrogel system (Figure S2). In addition, both nanofiber systems exhibited similar viscoelastic characteristics, which suggested that any difference in biological activity of these systems would not be caused by the difference of mechanical features between peptide nanofiber scaffolds but due to the functional epitopes presented on the collagen-mimetic nanofibers (Figure 1D).

Peptide Nanofiber Systems Provided a Biocompatible Environment for rMSCs. The viability of MSCs that were cultured on the peptide nanofiber systems and on TCP 

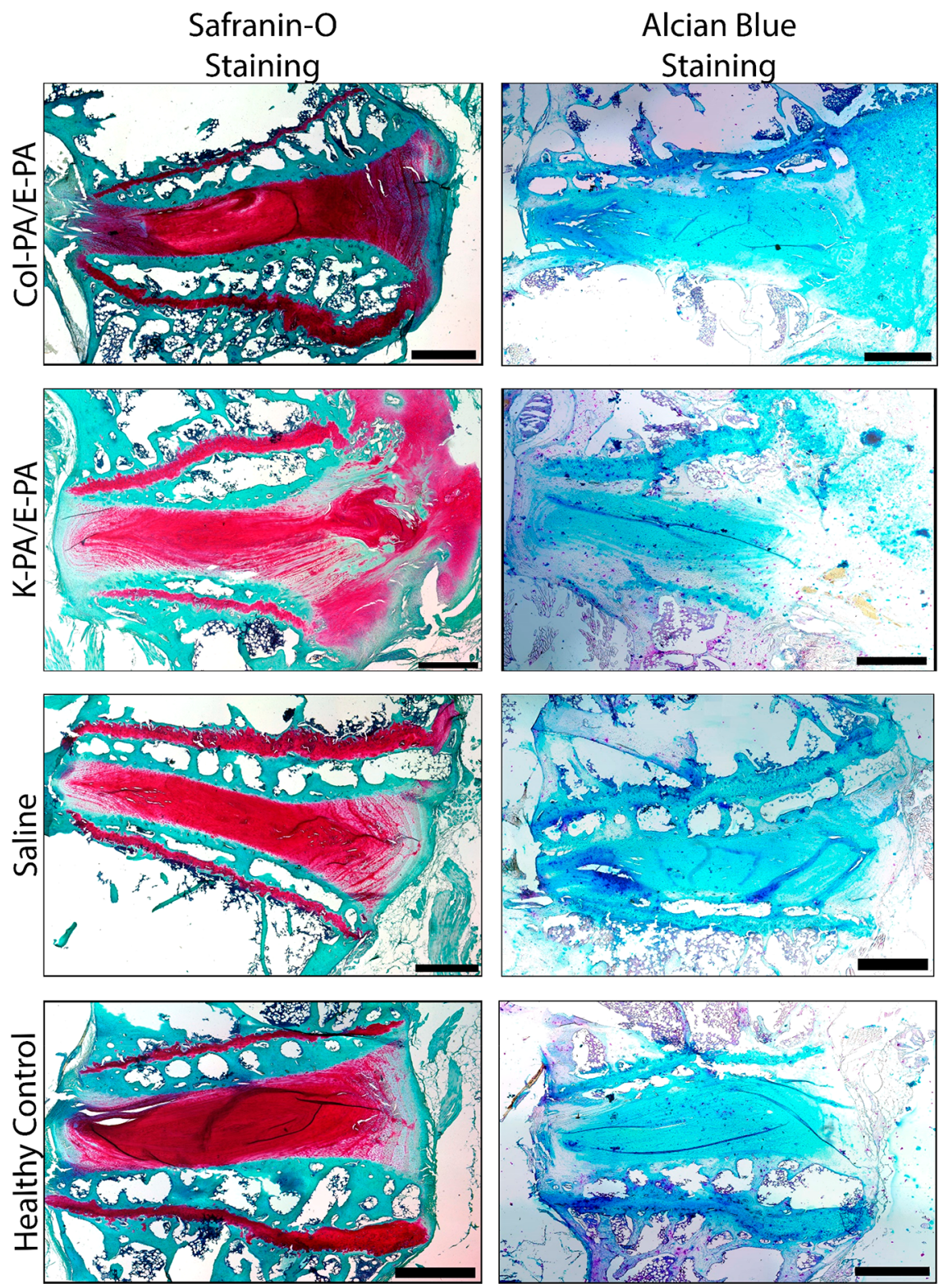

Figure 3. Glycosaminoglycan deposition analyses. Representative images of safranin-O/fast green/hematoxylin staining and Alcian blue staining for delayed degeneration groups (scale bars $=1 \mathrm{~mm}$ ). Note that glycosaminoglycan deposition was observed as intense red color in safranin-O staining images and observed as intense blue color in Alcian Blue staining images in NP and AF regions.

was evaluated by both Live/Dead Assay and alamarBlue Assay on three different culture days (day 1, day 2, and day 3) (Figure S3). In addition to MSCs, IVD cells isolated from rat IVDs were tracked for 5 days, and according to these results, IVD cells cultured on peptide nanofiber systems proliferated (Figure S4). Both of these experiments showed that the peptide nanofiber systems provided a suitable environment for cellular growth and that these scaffolds did not affect the viability of the MSCs and the IVD cells. Although the cells exhibited lower cellular metabolic activity on the peptide nanofiber scaffold systems than the TCP during first 2 days of culture in alamarBlue assay, they adapted to the environment on day 3. Since the Live/Dead assay showed that the peptide nanofiber scaffolds were not toxic to the cells, the reason behind the lower metabolic activity of cells on the peptide nanofibers measured by alamarBlue assay was attributed to the decreased number of proliferating cells, which was one of the indicators of differentiation. ${ }^{28}$
Collagen Peptide Presenting Nanofibers Induced Chondrogenic Differentiation of MSCs in Vitro. We investigated the chondrogenic differentiation potential of MSCs on collagen peptide presenting and control nanofibers due to their potential to be used in IVD regeneration. ${ }^{17}$ MSCs can differentiate into multiple lineages such as osteocytes, chondrocytes, and adipocytes, ${ }^{29,30}$ and cells that commit into chondrogenic lineage form cell aggregates by increasing their interactions and deposit glycosaminoglycans. ${ }^{31,32}$ Cells that were cultured on collagen peptide presenting nanofibers were observed to form more aggregates than the control groups. In addition, safranin-O staining showed that the cells cultured on the Col-PA/E-PA nanofiber system deposited more glycosaminoglycans than the other groups (Figure 2A).

Morphological changes of MSCs and safranin-O staining results were also confirmed by qRT-PCR experiments to quantify the expression level of Sox9, which is one of the most important early stage chondrogenic differentiation markers. ${ }^{33}$ 

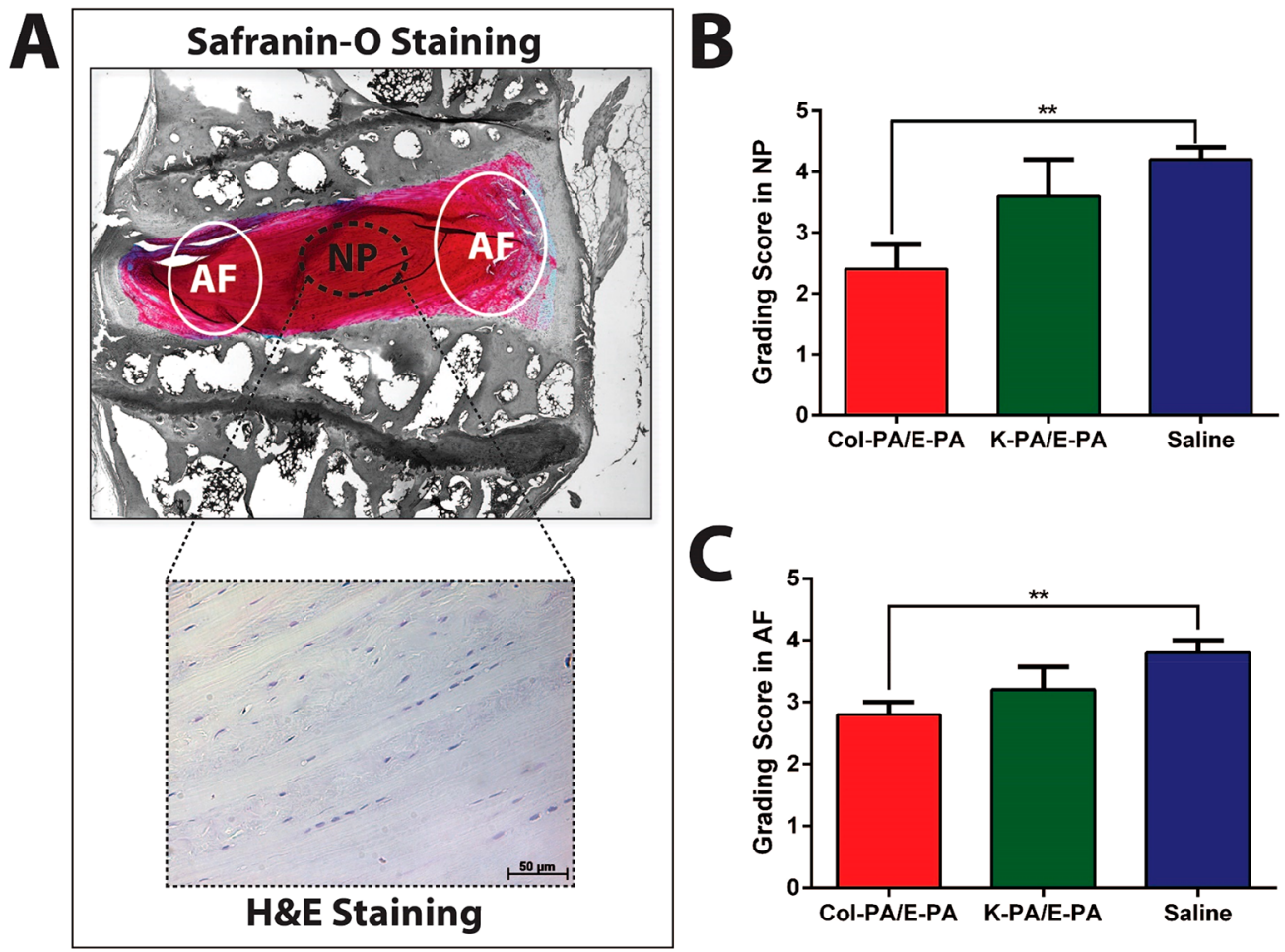

Figure 4. Analyses for degenerative changes in NP and AF. (A) Schematic representation of the scoring method. (B) Grading scores in NP for delayed degeneration groups through hematoxylin and eosin staining. (C) Grading scores in AF for delayed degeneration groups through safranin$\mathrm{O}$ staining ( 5 and 0 are maximum and minimum degeneration scores, respectively) ( $* * p=0.0025$ for NP scoring and $* * p=0.0077$ for AF scoring. Col-PA/E-PA group was compared with the saline group and K-PA/E-PA group separately by scoring with Student's $t$ test, mean \pm s.e.m.).

Sox9 has an important role in chondrogenic differentiation since Sox9 is required to express chondrogenic genes such as aggrecan and collagen type-II, which are necessary for the cartilage structure and function. ${ }^{30}$ On day 14 , the expression level of Sox9 in cells cultured on collagen peptide presenting nanofibers reached its greatest value and exhibited significantly increased Sox 9 expression by 2.7 -fold and 5.5-fold compared to K-PA/E-PA and TCP groups, respectively (Figure 2C). Cells cultured on TCP expressed high levels of Sox9 on day 7, which is probably due to the effect of chondrogenic media; however, cell cultured on Col-PA/E-PA nanofibers still had slightly higher gene expression level, which demonstrated the higher potential of initial chondrogenic differentiation induction of collagen peptide presenting nanofibers (Figure 2B).

Collagen Peptide Presenting Nanofiber Scaffold Induced Regeneration of Defected IVD Tissue in Rabbit Needle Puncture Model. IVD degeneration is characterized by natural ECM degradation, loss of water content of the tissue, and decreased cellular viability. ${ }^{34-36}$ An in vivo model of IVD degeneration was generated by puncturing the IVD tissue with a $18 \mathrm{G}$ needle in rabbits. Similar to the human IVD degeneration, both loss of integrity of ECM and decrease in disc height were achieved with this leakage, which shows the suitability of this model (Figure S5). Both early and late stages of IVD degeneration were modeled by establishing two experimental groups: delayed degeneration (scaffold injection after 2 weeks from the degeneration) and immediate degeneration (scaffold injection immediately after the degeneration). These experimental models enabled assessment of both protective and healing capacity of Col-PA/E-PA.

The grading scale described by Nagae et al. ${ }^{27}$ was used for the evaluation of IVD tissue regeneration, and it is briefly described in Table S2. The tissue sections were stained with safranin-O dye for the detection of the glycosaminoglycans (Figure 3). In addition, sulfated glycoproteins were detected with Alcian blue staining (Figure 3). In addition to the delayed degeneration groups, the immediate degeneration groups were also stained with safranin-O and Alcian blue staining (Figure S6). Both staining results demonstrated that the tissue sections from the Col-PA/E-PA peptide nanofiber treatment group showed the highest glycosaminoglycan content observed as intense red color in safranin-O staining images and observed as intense blue color in Alcian Blue staining images in NP and AF region compared to control peptide group and the saline group (Figure 3).

Cellular content of NP was analyzed by hematoxylin and eosin staining, which revealed the degenerative changes in NP with respect to the architecture of the proliferative connective tissue and cellular content of the tissue. The appearance of AF was graded according to the degree of appearance of lamellar structure and rupture through analysis of safranin-O stained sections (Figure 4A). The representative images for hematoxylin and eosin staining results for delayed degeneration groups are shown in Figure S7. Similar structures in Col-PA/E-PA and healthy control were observed in contrast to disorganized ECM structure in the saline group in Figure S7.

The NP degeneration scoring showed that the Col-PA/E-PA group had the lowest degeneration scores and this treatment provided effective regeneration, which was significantly better than the saline group. In addition, degeneration scores of the Col-PA/E-PA peptide nanofiber were lower than the control nanofiber (K-PA/E-PA) group. However, even the control nanofiber treated group had lower degeneration scores than the sham group probably because of the mechanical support to 

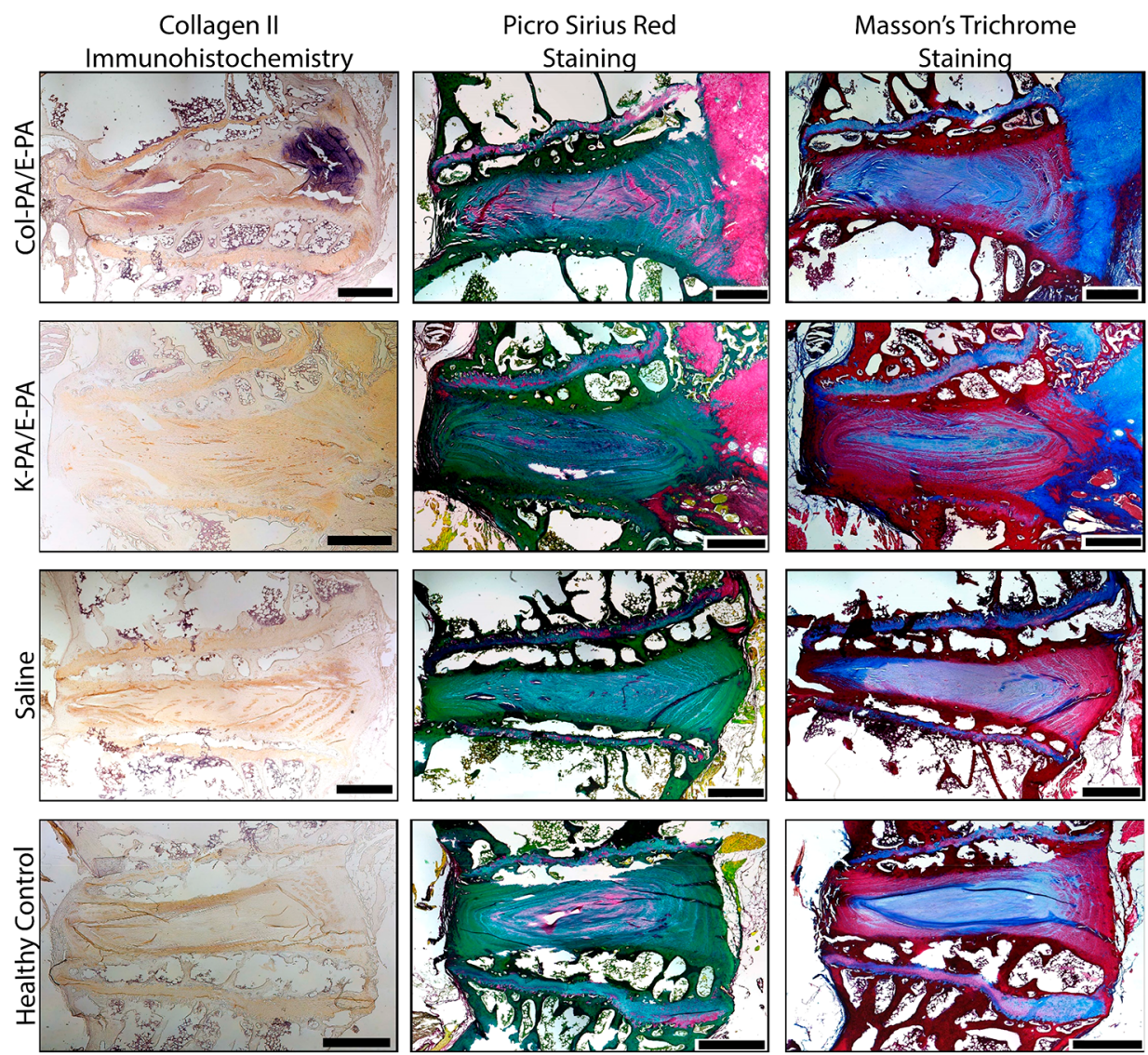

Figure 5. Collagen deposition analyses. Representative images of Collagen type-II immunostaining, Picro Sirius red staining, and Masson's trichrome staining for delayed degeneration groups (scale bars $=1 \mathrm{~mm}$ ). Note that collagen deposition were observed as brown in IHC staining, as red in Picro Sirius Staining, and as blue in Masson's trichrome staining.

the tissue provided by the peptide nanofiber systems (Figure 4B). A similar trend was also observed in the AF degeneration scoring result, which showed that the collagen peptide presenting nanofiber scaffold presented a biocompatible microenvironment and supportive mechanical properties for the $\mathrm{AF}$ cells, and it sustained their proliferation and differentiation activities (Figure 4C). It is important to note that the degeneration scoring results of the immediate degeneration groups demonstrated that Col-PA/E-PA nanofiber scaffold also significantly prevented the further progression of IVD degeneration (Figures S8 and S9).

Collagen type-II immunostaining demonstrated that the cells in Col-PA/E-PA treated tissues deposited higher amounts of collagen type-II than the other groups (Figure 5). In addition, Picro Sirius Red and Masson's trichrome stainings were also performed to detect the collagen deposition in the tissues. According to the results of both Picro Sirius red and Masson's trichrome stainings, the Col-PA/E-PA treated animals had the highest collagen content observed as pink color in Picro Sirius red staining images ${ }^{37}$ and observed as blue color in Masson's trichrome staining images. It should be noted that since the collagen mimetic epitopes are presented on the peptide nanofiber scaffold as anchored molecules and not as soluble ones, collagen stainings in these results are truly the consequence of newly synthesized collagen molecules as one of the signs of IVD tissue regeneration. They had very similar appearance with healthy control tissues regarding both collagen content and general structural properties, which further demonstrated that collagen peptide presenting nano- fibers also maintained the collagen structure of tissue, which is essential for the proper functioning of the tissue. ${ }^{38}$

\section{DISCUSSION}

LBP is one of the most common spinal problems throughout the world because of the increase in the requirement for spending longer time at a desk for work. ${ }^{37,39}$ Despite its economic and health consequences, the current treatment strategies for LBP mostly aim to reduce the pain. For the effective treatment of LBP associated with IVD, the ideal treatment strategies should include maintenance of cellular components of the tissue and induction of differentiation of chondrocyte-like cells to the NP and AF cells to promote ECM production ${ }^{13}$ because when the ECM of the IVD cannot be regenerated properly, tissue degeneration continues to progress more severely. ECM mimetic hydrogel scaffolds that have a porous structure and high hydration capacity can also provide efficient alternative treatment options. Especially at the early stages of the disease, effective tissue engineering methods using these hydrogels can aid the recovery of the tissue. In this study, we used a collagen peptide presenting nanofiber scaffold to enhance IVD regeneration, where we investigated its effects on the viability and differentiation of MSCs into chondrocytes in vitro and maintenance of tissue structure and macromolecular content after IVD degeneration in vivo.

Since IVD degeneration is characterized by a decrease in the number of functional and viable cells, the proliferation of the cells that are already residing in the tissue and the recruitment of other cells from the surrounding environment is important 
for the regeneration process. For this purpose, stem cell therapy is one of the promising treatment options of IVD degeneration. ${ }^{40}$ However, since the tissue microenvironment is also vital for the newly proliferated and recruited cells, induction of ECM remodeling via cell carrier scaffolds as ECM substitutes should also be considered besides stem cell therapies. In addition to act as a supportive environment for the transplanted cells, biomaterial applications are also very important for mechanical support, improving disc height, affecting gene transcription and ECM biosynthesis. NP cells or MSCs embedded in hydrogels have been shown to induce expression of genes required for ECM remodeling when they were cultured in chitosan-based hydrogels ${ }^{41,42}$ or in type II collagen gels stabilized with PEG and hyaluronan. ${ }^{43}$ Hence, we also investigated the biocompatibility of the Col-PA/E-PA nanofiber system for MSCs as an ECM mimetic scaffold as these cells are the most widely used cells for implantation. The Col-PA/E-PA nanofibers provided a biocompatible environment and induced differentiation of MSCs into chondrocytes suggesting that the collagen peptide presenting nanofiber scaffold can be considered for further stem cell transplantation applications. $^{44}$

On the other hand, even though cell-based approaches have several advantages, they often require two-step procedures because of the need to harvest cells in the first surgery. Therefore, bioactive cell-free scaffolds have the advantage to enable a one-step treatment procedure and to avoid other time-consuming technical difficulties for cell culture. To investigate the effectiveness of the Col-PA/E-PA nanofiber scaffold as an ECM substitute for IVD regeneration, an in vivo model of rabbit IVD degeneration was utilized. Both early and late stages of the degeneration were modeled by using two different experimental groups: delayed degeneration group (gel injection 2 weeks after the degeneration process) and immediate degeneration group (gel injection immediately after the degeneration process) (Table S1). After modeling the delayed and immediate degeneration processes of IVD degeneration, IVD tissues were extracted 4 weeks after gel injection. This time point was chosen according to the literature and the results of our own optimization studies, which were performed to determine the proper time period and working gel concentration, and the proper surgical model for IVD degeneration and regeneration. ${ }^{45}$ Since the IVD degeneration in humans progress slowly rather than an effect of a sudden injury, both immediate and delayed degeneration models were utilized to see the effect of the collagen peptide presenting scaffold on the IVD regeneration process. By using the immediate degeneration model, it was aimed to analyze whether the progression of the IVD degeneration can be prevented with gel injection. On the other hand, the delayed degeneration model was utilized to analyze whether collagen peptide presenting nanofiber scaffold is able to stop the degeneration process and help the regeneration of the IVD tissue, even if the treatment has been delayed for a while. The histochemical analyses of the delayed degeneration group showed that the Col-PA/E-PA nanofiber scaffold exhibited significant healing capacity and provided better IVD morphology compared to the control nanofiber system and saline-treated groups. In addition, glycosaminoglycan and collagen deposition and expression level of collagen type-II on the tissues that were treated with Col-PA/E-PA nanofibers were observed to increase compared to nonbioactive controls, and similar collagen content and comparable appearance with the healthy control tissue were revealed by Picro Sirius and Masson's trichrome stainings. We also observed a significant decrease in degeneration score in delayed degeneration group for Col-PA/E-PA treated tissues compared to the salinetreated tissues.

For immediate degeneration groups, a statistically significant difference between Col-PA/E-PA treated tissues and salinetreated tissues was also detected but not as high as delayed degeneration groups. This may be caused by the degeneration duration used in this model not being adequate for enough degeneration to occur. However, these findings demonstrated that the collagen peptide presenting bioactive nanofiber scaffold provides an effective treatment strategy for both early and late stages of IVD degeneration to prevent the disease symptoms and also to ameliorate disease progression in the late stages of degeneration.

Collagen is one of the major components of the healthy IVD tissue. Collagen fibers function as a scaffold for the cells and provide mechanical strength and water holding capacity to the tissue. However, the application range of native collagen is limited due to immunogenic or pathological risks and drawbacks associated with collagen's physical characteristics (weak scaffold with low mechanical strength, shrinkage problem, batch-to-batch variation, and large porosity). Hence, the collagen-like structures are useful for obtaining efficient tissue regeneration therapeutics. Therefore, in this study, the collagen peptide presenting nanofibers were developed to mimic the natural collagen function by supporting the generation of natural lamellar structure of AF and glycosaminoglycan deposition in NP. Since the mechanical properties of the peptide amphiphile scaffolds are greater than the natural collagen gels (Figure 1D), injection of the peptide nanofibers can enhance the mechanical properties of degenerated IVD tissue. Through the self-assembly feature of the peptide amphiphile molecules, the bioactive sequence of natural collagen molecules (POG) was presented on the surface of the nanofiber structures. Together with its specific functional bioactivity, mechanical properties of peptide nanofibers make Col-PA/E-PA an effective therapeutic agent for IVD regeneration. Our findings overall suggest that proliferation and differentiation of NP cells during IVD regeneration were supported by the collagen peptide presenting bioactive nanofiber scaffold. Furthermore, immunohistochemical and scoring results for the degenerative changes in the tissue structure demonstrated that without any additional growth factors or cell implantation, collagen-mimetic bioactive peptide nanofibers showed great regeneration capacity in the IVD tissue.

\section{CONCLUSION}

LBP is mostly associated with IVD degeneration and it is one of the most common spinal problems throughout the world. Therefore, developing effective treatment strategies is necessary to both relieve the patient from severe pain and also to induce regeneration of the tissue. In this study, we investigated the in vitro potential of Col-PA/E-PA nanofiber scaffold for the differentiation of MSCs into chondrocytes and the efficiency of this bioactive scaffold for restoring damaged IVD function in an in vivo model of rabbit IVD degeneration. We observed that the collagen peptide presenting nanofiber scaffold provided a biocompatible environment for MSCs, enabled their differentiation into chondrocytes, and also preserved the IVD morphology and function in vivo. Overall, this bioactive 
collagen peptide presenting nanofiber scaffold could prevent the progression of IVD degeneration and provide further functional recovery of the tissue for IVD treatment in clinical applications.

\section{ASSOCIATED CONTENT}

\section{S Supporting Information}

The Supporting Information is available free of charge on the ACS Publications website at DOI: 10.1021/acsabm.9b00062.

LC-MS results, TEM images of peptide nanofiber systems, viability analyses of rMSCs, in vivo IVD degeneration model, additional histological staining figures and scoring results (PDF)

\section{AUTHOR INFORMATION}

\section{Corresponding Authors}

*E-mail: sukrucaglar983@gmail.com.

*E-mail: mguler@uchicago.edu.

*E-mail: atekinay@bilkent.edu.tr.

\section{ORCID}

Mustafa O. Guler: 0000-0003-1168-202X

Ayse B. Tekinay: 0000-0002-4453-814X

\section{Author Contributions}

O.U., E.A., Y.S.C., M.O.G., and A.B.T. designed the study. O.U., E.A., and G.G. performed research. O.U., E.A., M.C.K., H.O., and I.D. performed the animal experiments and histology analyses. O.U. and E.A. analyzed data. Y.S.C., M.O.G., and A.B.T. advised and analyzed the research. O.U., E.A., M.O.G., and A.B.T. wrote the paper with contributions of all the authors. All authors have given approval to the final version of the manuscript.

\section{Notes}

The authors declare no competing financial interest.

\section{ACKNOWLEDGMENTS}

We thank Dr. A. D. Ozkan for helpful comments. TOC figure was prepared using Mind the Graph software. A.B.T. thanks the Science Academy Award (BAGEP).

\section{ABBREVIATIONS}

LBP, lower back pain; IVD, intervertebral disc; NP, nucleus pulposus; AF, annulus fibrosus; PA, peptide amphiphile; ECM, extracellular matrix; $C D$, circular dichroism; SEM, scanning electron microscopy; HPLC, high performance liquid chromatography; s.e.m., standard error of mean; LC-MS, liquid chromatography-mass spectroscopy.

\section{REFERENCES}

(1) Rubin, D. I. Epidemiology and Risk Factors for Spine Pain. Neurol. Clin. 2007, 25, 353-371.

(2) Sato, M.; Asazuma, T.; Ishihara, M.; Kikuchi, T.; Kikuchi, M.; Fujikawa, K.; et al. An Experimental Study of the Regeneration of the Intervertebral Disc with an Allograft of Cultured Annulus Fibrosus Cells Using a Tissue-engineering Method. Spine (Philadelphia) 2003, $28,548-553$.

(3) McBeth, J.; Jones, K. Epidemiology of Chronic Musculoskeletal Pain. Best. Pract. Res. Clin. Rheumatol. 2007, 21, 403-425.

(4) Huang, Y. C.; Urban, J. P.; Luk, K. D. Intervertebral Disc Regeneration: Do Nutrients Lead the Way? Nat. Rev. Rheumatol. 2014, 10, 561-566.

(5) Samartzis, D.; Karppinen, J.; Chan, D.; Luk, K. D.; Cheung, K. M. The Association of Lumbar Intervertebral Disc Degeneration on
Magnetic Resonance Imaging with Body Mass Index in Overweight and Obese Adults: a Population-based Study. Arthritis Rheum. 2012, 64, 1488-1496.

(6) Yu, J.; Winlove, P. C.; Roberts, S.; Urban, J. P. Elastic Fibre Organization in the Intervertebral Discs of the Bovine Tail. J. Anat. 2002, 201, 465-475.

(7) Inoue, H. Three-dimensional Architecture of Lumbar Intervertebral Discs. Spine (Philadelphia) 1981, 6, 139-146.

(8) Marchand, F.; Ahmed, A. M. Investigation of the Laminate Structure of Lumbar Disc Anulus Fibrosus. Spine (Philadelphia) 1990, 15, 402-410.

(9) Errington, R. J.; Puustjarvi, K.; White, I. R.; Roberts, S.; Urban, J. P. Characterisation of Cytoplasm-Filled Processes in Cells of the Intervertebral Disc. J. Anat. 1998, 192, 369-378.

(10) Bruehlmann, S. B.; Rattner, J. B.; Matyas, J. R.; Duncan, N. A. Regional Variations in the Cellular Matrix of the Annulus Fibrosus of the Intervertebral Disc. J. Anat. 2002, 201, 159-171.

(11) Humzah, M.D. R.W.; et al. Soames; Human Intervertebral Disc: Structure and Function. Anat. Rec. 1988, 220, 337-356.

(12) Freburger, J. K.; Holmes, G. M.; Agans, R. P.; Jackman, A. M.; Darter, J. D.; Wallace, A. S.; Castel, L. D.; Kalsbeek, W. D.; Carey, T. S. The Rising Prevalence of Chronic Low Back Pain. Arch. Intern. Med. 2009, 169, 251-258.

(13) Mochida, J.; Nishimura, K.; Nomura, T.; Toh, E.; Chiba, M. The Importance of Preserving Disc Structure in Surgical Approaches to Lumbar Disc Herniation. Spine (Philadelphia) 1996, 21, 15631564.

(14) Siepe, C. J.; Heider, F.; Wiechert, K.; Hitzl, W.; Ishak, B.; Mayer, M. H. Mid- to Long-term Results of Total Lumbar Disc Replacement: A Prospective Analysis with 5- to 10-Year Follow-up. Spine. J. 2014, 14, 1417-1431.

(15) Alini, M.; Li, W.; Markovic, P.; Aebi, M.; Spiro, R. C.; Roughley, P. J. The Potential and Limitations of a Cell-seeded Collagen/Hyaluronan Scaffold to Engineer an Intervertebral Disc-like Matrix. Spine (Philadelphia) 2003, 28, 446-454.

(16) O'Halloran, D. M.; Pandit, A. S. Tissue-engineering Approach to Regenerating the Intervertebral Disc. Tissue Eng. 2007, 13, 19271954.

(17) Raj, P. P. Intervertebral Disc: Anatomy-Physiology-Pathophysiology-Treatment. Pain. Pract. 2008, 8, 18-44.

(18) Arslan, E.; Garip, I. C.; Gulseren, G.; Tekinay, A. B.; Guler, M. O. Bioactive Supramolecular Peptide Nanofibers for Regenerative Medicine. Adv. Healthcare Mater. 2014, 3, 1357-1376.

(19) Eren Cimenci, C.; Uzunalli, G.; Uysal, O.; Yergoz, F.; Karaca Umay, E.; Guler, M. O.; Tekinay, A. B. Laminin Mimetic Peptide Nanofibers Regenerate Acute Muscle Defect. Acta Biomater. 2017, 60, 190-200.

(20) Mammadov, R.; Mammadov, B.; Toksoz, S.; Aydin, B.; Yagci, R.; Tekinay, A. B.; Guler, M. O. Heparin Mimetic Peptide Nanofibers Promote Angiogenesis. Biomacromolecules 2011, 12, 3508-3519.

(21) Mammadov, B.; Mammadov, R.; Guler, M. O.; Tekinay, A. B. Cooperative Effect of Heparan Sulfate and Laminin Mimetic Peptide Nanofibers on the Promotion of Neurite Outgrowth. Acta Biomater. 2012, 8, 2077-2086.

(22) Kocabey, S.; Ceylan, H.; Tekinay, A. B.; Guler, M. O. Glycosaminoglycan Mimetic Peptide Nanofibers Promote Mineralization by Osteogenic Cells. Acta Biomater. 2013, 9, 9075-85.

(23) Lee, S. S.; Hsu, E. L.; Mendoza, M.; Ghodasra, J.; Nickoli, M. S.; Ashtekar, A.; Polavarapu, M.; Babu, J.; Riaz, R. M.; Nicolas, J. D.; Nelson, D.; Hashmi, S. Z.; Kaltz, S. R.; Earhart, J. S.; Merk, B. R.; McKee, J. S.; Bairstow, S. F.; Shah, R. N.; Hsu, W. K.; Stupp, S. I. Gel Scaffolds of BMP-2-binding Peptide Amphiphile Nanofibers for Spinal Arthrodesis. Adv. Healthcare Mater. 2015, 4, 131-141.

(24) Tysseling, V. M.; Sahni, V.; Pashuck, E. T.; Birch, D.; Hebert, A.; Czeisler, C.; Stupp, S. I.; Kessler, J. A. Self-assembling Peptide Amphiphile Promotes Plasticity of Serotonergic Fibers Following Spinal Cord Injury. J. Neurosci. Res. 2010, 88, 3161-3170. 
(25) Nagarajan, V.; Kamitori, S.; Okuyama, K. Structure Analysis of a Collagen-Model Peptide with a (Pro-Hyp-Gly) Sequence Repeat. J. Biochem. 1999, 125, 310-318.

(26) Lee, J. T.; Cheung, K. M.; Leung, V. Y. Systematic Study of Cell Isolation from Bovine Nucleus Pulposus: Improving Cell Yield and Experiment Reliability. J. Orthop. Res. 2015, 33, 1743-1755.

(27) Nagae, M.; Ikeda, T.; Mikami, Y.; Hase, H.; Ozawa, H.; Matsuda, K.; Sakamoto, H.; Tabata, Y.; Kawata, M.; Kubo, T. Intervertebral Disc Regeneration Using Platelet-rich Plasma and Biodegradable Gelatin Hydrogel Microspheres. Tissue Eng. 2007, 13, 147-158.

(28) Cooper, G. M. Cell Proliferation in Development and Differentiation. The Cell: A Molecular Approach; 2nd ed.; Sinauer Associates: Sunderland, MA, 2000.

(29) Pittenger, M. F.; Mackay, A. M.; Beck, S. C.; Jaiswal, R. K.; Douglas, R.; Mosca, J. D.; Moorman, M. A.; Simonetti, D. W.; Craig, S.; Marshak, D. R. Multilineage Potential of Adult Human Mesenchymal Stem Cells. Science 1999, 284, 143-147.

(30) Jiang, Y.; Jahagirdar, B. N.; Reinhardt, R. L.; Schwartz, R. E.; Keene, C. D.; Ortiz-Gonzalez, X. R.; Reyes, M.; Lenvik, T.; Lund, T.; Blackstad, M.; Du, J.; Aldrich, S.; Lisberg, A.; Low, W. C.; Largaespada, D. A.; Verfaillie, C. M. Pluripotency of Mesenchymal Stem Cells Derived from Adult Marrow. Nature 2002, 418, 41-49.

(31) Hall, B. K.; Miyake, T. Divide; Accumulate; Differentiate: Cell Condensation in Skeletal Development Revisited. Int. J. Dev. Biol. 1995, 39, 881-893.

(32) Goldring, M. B.; Tsuchimochi, K.; Ijiri, K. The Control of Chondrogenesis. J. Cell. Biochem. 2006, 97, 33-44.

(33) Akiyama, H. Control of Chondrogenesis by the Transcription Factor Sox9. Mod. Rheumatol. 2008, 18, 213-219.

(34) Lyons, G.; Eisenstein, S. M.; Sweet, M. B. Biochemical Changes in Intervertebral Disc Degeneration. Biochim. Biophys. Acta, Gen. Subj. 1981, 673, 443-453.

(35) Adams, M. A.; Freeman, B. J.; Morrison, H. P.; Nelson, I. W.; Dolan, P. Mechanical Initiation of Intervertebral Disc Degeneration. Spine (Philadelphia) 2000, 25, 1625-1636.

(36) Roughley, P. J. Biology of Intervertebral Disc Aging and Degeneration: Involvement of the Extracellular Matrix. Spine (Philadelphia) 2004, 29, 2691-2699.

(37) Vogel, B.; Siebert, H.; Hofmann, U.; Frantz, S. Determination of Collagen Content within Picrosirius Red Stained ParaffinEmbedded Tissue Sections Using Fluorescence Microscopy. MethodsX 2015, 2, 124-134.

(38) Zhao, C. Q.; Wang, L. M.; Jiang, L. S.; Dai, L. Y. The Cell Biology of Intervertebral Disc Aging and Degeneration. Ageing Res. Rev. 2007, 6, 247-261.

(39) Buckwalter, J. A. Aging and Degeneration of the Human Intervertebral Disc. Spine (Philadelphia) 1995, 20, 1307-1314.

(40) Sakai, D.; Andersson, G. B. Stem Cell Therapy for Intervertebral Disc Regeneration: Obstacles and Solutions. Nat. Rev. Rheumatol. 2015, 11, 243-256.

(41) Cheng, Y. H.; Yang, S. H.; Lin, F. H. Thermosensitive Chitosan-Gelatin-Glycerol Phosphate Hydrogel as a Controlled Release System of Ferulic Acid for Nucleus Pulposus Regeneration. Biomaterials 2011, 32, 6953-6961.

(42) Cheng, Y. H.; Yang, S. H.; Su, W. Y.; Chen, Y. C.; Yang, K. C.; Cheng, W. T.; Wu, S. C.; Lin, F. H. Thermosensitive ChitosanGelatin-Glycerol Phosphate Hydrogels as a Cell Carrier for Nucleus Pulposus Regeneration: an in vitro Study. Tissue Eng., Part A 2010, 16, 695-703.

(43) Collin, E. C.; Grad, S.; Zeugolis, D. I.; Vinatier, C. S.; Clouet, J. R.; Guicheux, J. J.; Weiss, P.; Alini, M.; Pandit, A. S. An Injectable Vehicle For Nucleus Pulposus Cell-based Therapy. Biomaterials 2011, 32, 2862-2870.

(44) Vadalà, G.; Sowa, G.; Hubert, M.; Gilbertson, L. G.; Denaro, V.; Kang, J. D. Mesenchymal Stem Cells Injection in Degenerated Intervertebral Disc: Cell Leakage may Induce Osteophyte Formation. J. Tissue Eng. Regener. Med. 2012, 6, 348-355.
(45) Masuda, K.; Aota, Y.; Muehleman, C.; Imai, Y.; Okuma, M.; Thonar, E. J.; Andersson, G. B.; An, H. S. A Novel Rabbit Model of Mild; Reproducible Disc Degeneration by an Anulus Needle Puncture: Correlation between the Degree of Disc Injury and Radiological and Histological Appearances of Disc Degeneration. Spine (Philadelphia) 2005, 30, 5-14. 